\title{
Development and Administration of Chronic Suppurative Otitis Media Questionnaire-12 (COMQ-12) and Chronic Otitis Media Outcome Test-15 (COMOT-15) in Kannada
}

\author{
Prashanth Prabhu $^{1}$ Anusha Chandrashekar ${ }^{1} \quad$ Anita Jose $^{1} \quad$ Aishwarya Ganeshan $^{1}$ Lavanya Kiruthika ${ }^{1}$ \\ ${ }^{1}$ Department of Audiology, All India Institute of Speech and Hearing, \\ Mysore, Karnataka, India \\ Int Arch Otorhinolaryngol 2018;22:108-112. \\ Address for correspondence Prashanth Prabhu, Department of \\ Audiology, All India Institute of Speech and Hearing, Naimisham \\ Campus Manasagangothri, Mysore, Karnataka 570006, India \\ (e-mail: prashanth.audio@gmail.com).
}

\begin{abstract}
Introduction It is essential to determine the quality of life among individuals with chronic suppurative otitis media (CSOM). However, there is limited literature on healthrelated quality of life measurements in Kannada-speaking individuals with CSOM. Objectives The present study attempted to translate and validate Chronic Suppurative Otitis Media Questionnaire-12 (COMQ-12) and Chronic Otitis Media Outcome Test15 (COMOT-15) in Kannada.

Method The English questionnaires were translated and back-translated, and they were later verified for content validity. The developed questionnaires were then administered to 100 individuals with CSOM for further validation.

Results The results of the study showed that the translated questionnaires have good internal consistency for measuring quality of life among individuals with CSOM. The study also showed that Kannada-speaking individuals with CSOM show significant

Keywords

- chronic suppurative otitis media questionnaire

- translation

- quality of life impairment in their health-related quality of life measures.

Conclusions The impairments were more related to ear symptoms and psychological issues. However, test-retest reliability of the developed questionnaire and its further validation are essential. Thus, these questionnaires attempt to understand the problems of the individuals with CSOM from the patients' perspective and help clinicians provide the appropriate management.
\end{abstract}

\section{Introduction}

Otitis media $(\mathrm{OM})$ is an umbrella term for a cluster of complex infectious and inflammatory conditions affecting the middle ear. It involves pathologies of the middle ear and middle ear mucosa. There are various subtypes of OM, such as acute otitis media (AOM), otitis media with effusion (OME), chronic suppurative otitis media (CSOM), mastoiditis, and cholesteatoma. Chronic suppurative otitis media (CSOM) is defined as a chronic infection of the middle ear cleft with or without perforation. ${ }^{1}$ It is one of the subtypes of $\mathrm{OM}$, and it is a long-standing suppurative middle ear cleft inflammation. The incidence

received

December 13, 2016

accepted

April 1, 2017

published online

June 5, 2017

$10.1055 / \mathrm{s}-0037-1603644$. ISSN $1809-9777$. rate of CSOM is $4.76 \%$, equating to 31 million cases, with $22.6 \%$ of cases occurring annually in children under the age of $5,50 \%$ of which suffer from hearing impairment. ${ }^{2}$ A World Health Organization (WHO) report suggests that 65 to 350 million individuals are suffering from CSOM globally, and the disease is a leading cause of hospital visits. ${ }^{2}$ This burden falls disproportionately on children in developing countries. Chronic suppurative otitis media presents with a chronically draining ear for 4-6 weeks, with a possible history of recurrent AOM, traumatic perforation, or insertion of grommets; other symptoms may include otorrhea, otalgia or fever, vertigo,

Copyright @ 2018 by Thieme Revinter

Publicações Ltda, Rio de Janeiro, Brazil

License terms

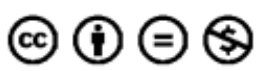


hearing loss and tinnitus. These symptoms associated with CSOM compromise the quality of life of the individuals with the disease. It affects social communication and professional life; another major symptom that stimulates social withdrawal is resistant malodorous ear drainage.

The health-related quality of life (HRQOL) measurement is considered an important health outcome in the clinical evaluation and healthcare research. ${ }^{3}$ Health-related quality of life is also used extensively in the clinical practice to help physclinicians make appropriate treatment decisions. Chronic suppurative otitis media is reported to affect $\sim 2 \%$ of the population globally, ${ }^{1}$ and $7.8 \%$ of the population of India. ${ }^{2}$ It is associated with hearing loss, which results in communication difficulties that affect social interactions and the professional life. In individuals with a higher degree of hearing loss, withdrawal from social activities may be observed. ${ }^{4,5}$ The presence of ear discharge is also a common reason for social withdrawal that affects the quality of life of individuals with CSOM. ${ }^{6}$

Chronic Otitis Media Questionnaire-12 (COMQ-12), developed by Philips, Haggard and Yung, ${ }^{7}$ and Chronic Otitis Media Outcome Test-15 (COMOT-15), developed by Baumann et al, ${ }^{8}$ are standardized outcome measures used to determine the HRQOL in individuals with CSOM. AWHO report shows a high prevalence of CSOM, especially in Southern India. ${ }^{2}$ Kannada is one of the major Southern Indian languages, with 50.8 million native speakers. Chronic suppurative otitis media is also reported to be more prevalent among the rural population, ${ }^{2}$ and the translation of the HRQOL measure into their native language is warranted. Hence, the present study has attempted to develop and validate COMQ-12 and COMOT-15 in Kannada. It has also attempted to determine the internal consistency of the developed questionnaires. This would be beneficial in determining the quality of life changes caused by CSOM in native Kannada speakers. The study also attempted to determine the possible predictors based on demographic details (gender and onset of hearing loss) and pure tone average (PTA).

\section{Method}

Chronic Otitis Media Questionnaire-12 has 12 questions with a 6 -point scale rating. Eight questions ${ }^{1-7,9}$ are related to the severity of the complaints, and the other four questions ${ }^{8,10-12}$ are related to the frequency of acts concerning the ear problems. Similarly, the 15 questions of COMOT-15 consist of 3 subscales categorized as ear symptoms (questions 1-6), hearing function (questions 7-9), and mental health (questions 10-15). ${ }^{9,11-13}$ The Kannada version of COMQ-12 and COMOT-15 was obtained by translation and back-translation. ${ }^{10}$ The Kannada version was back-translated to English by four lecturers of the Kannada language. The translated form was given to ten native Kannada-speaking audiologists for content validation. They were asked to rate the items as appropriate or not-appropriate on a 5-point rating scale. The audiologists considered both cultural and language issues while rating the questions. The social and health-related aspects specific to the region were kept in mind during the process. The questions rated as appropriate were retained, and the others were reframed according to the appropriateness of the sen- tence. The questions that were grammatically correct and appropriate to the Indian culture were included. The procedure for translating the questionnaire is shown as a flow chart in -Fig. 1.

The translated COMQ-12 and COMOT-15 were administered to 80 individuals diagnosed with CSOM. The group included 45 males and 35 females with ages ranging from 18 to 44 years (mean $=27.8$ years, standard deviation [SD] $=7.22$ ). A total of 65 out of the 80 patients could read Kannada, and they filled the questionnaire on their own. The remaining 15 were illiterate: the clinician read out the questions to them, and their responses were scored. The diagnosis of CSOM was made by an otorhinolaryngologist. The degree of hearing loss ranged from minimal hearing loss to moderately severe hearing loss based on the Clark ${ }^{11}$ classification. If hearing loss was present, it was of the conductive type in all the participants of the study. The onset of the hearing loss reported ranged from 1 month to 8 months. The translated COMQ- 12 was administered similarly to the original procedure suggested by Philips, Haggard and Yung. ${ }^{7}$ The 12 questions were asked, and the participants were instructed to rate their answers on a 6-point rating scale in which each point means the following: 0- never; 1 - at least once every 3 months; 2 - at least once a month; 3- once a week, 4- many days a week; and 5- always. The

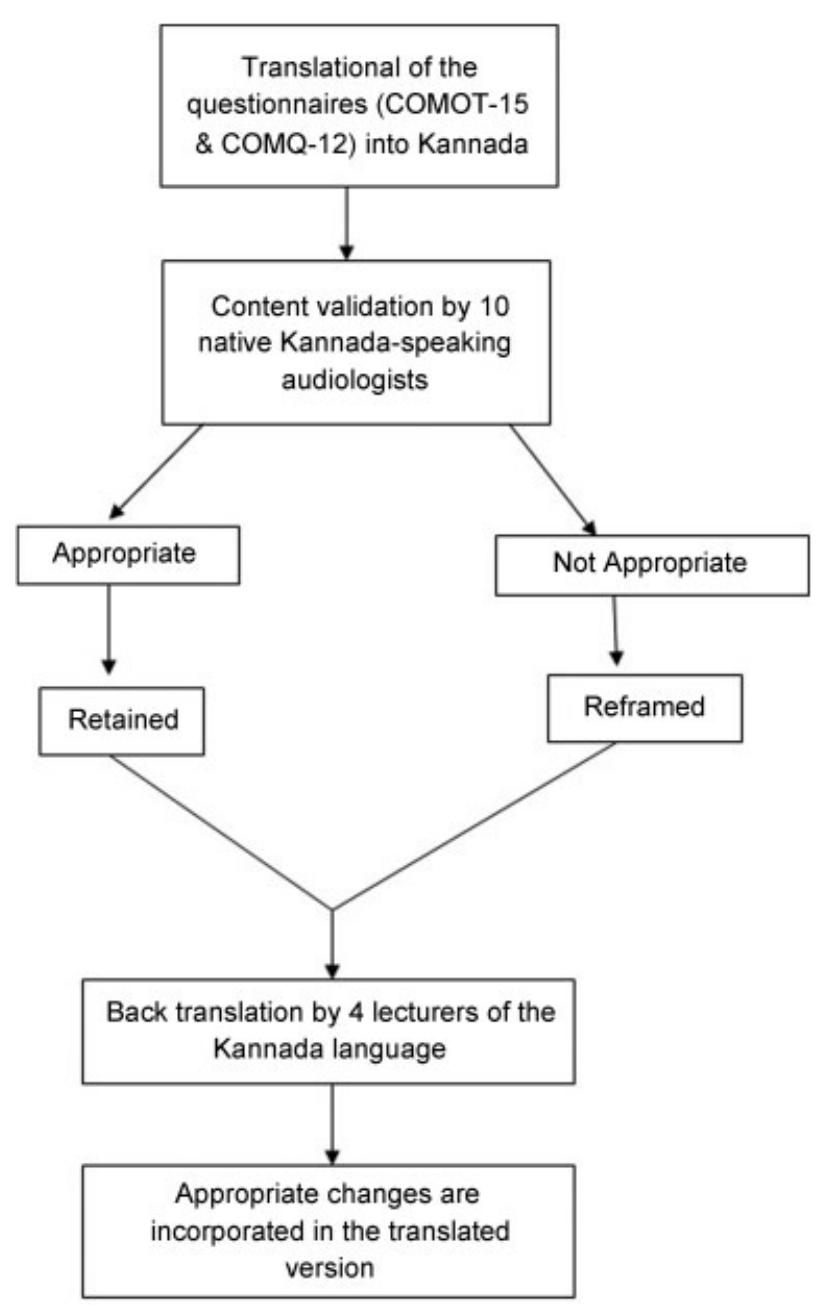

Fig. 1 The procedure used in translating the questionnaires. 


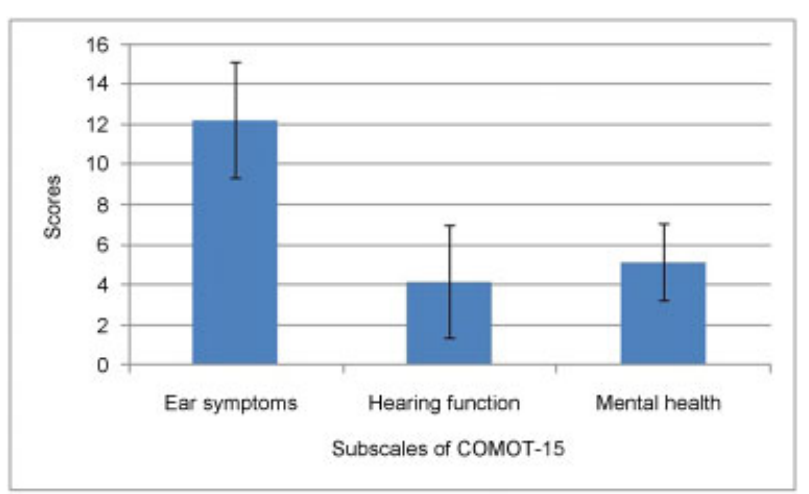

Fig. 2 Mean and SD of the scores obtained for the subscales of the Kannada version of COMOT-15.

translated COMOT-15 was also administered according to the original procedure recommended by Baumann et al. ${ }^{8}$ The participants had to answer 15 questions on a 6-point rating scale. The level of the problem was rated as: 0 - no problem at all; 1 - very small; 2 - small; 3- medium; 4- difficult; and 5 -very difficult. The 3 subscales of COMOT- 15 that assess the different dimensions (ear symptoms, hearing function and mental health) were recorded and analyzed. The internal consistency of the test was determined using Cronbach's $\alpha$, and the itemtotal correlation was also determined. A multiple regression analysis was performed to compare the predictors (PTA, gender, onset of the hearing loss and nature of the hearing loss) on the COMQ-12 and COMOT-15 scores.

\section{Ethical Considerations}

In the present study, all the testing procedures were performed using a non-invasive technique and adhering to the conditions of the ethical approval committee of the institute. All the test procedures were explained to the patients and their family members before testing, and informed consent was taken from all the patients or their family members for the participation in the study.

\section{Results}

The results of the study showed that the mean total score of COMQ-12 in Kannada was 24.11 (SD = 5.43), suggesting poor quality of life because of CSOM among the participants of the study. The mean score of COMOT-15 in Kannada was 23.8 ( $\mathrm{SD}=6.17$ ), which also suggests deterioration of health-related quality of life in individuals with CSOM. The mean scores on the subscales of COMOT-15 in Kannada were $12.23(\mathrm{SD}=2.9)$ for ear symptoms, $4.2(\mathrm{SD}=2.8)$ for hearing function, and 5.15 (SD = 1.9) for mental health, as shown in - Fig. 2. This suggests that ear symptoms were the major cause of reduced quality of life in Kannada-speaking individuals with CSOM.

The internal consistency was determined by Cronbach's $\alpha$ using the Statistical Package for the Social Sciences (SPSS, IBM Corp. Armonk, NY, US) software, version 21. The results showed that the Kannada version of COMQ-12 had an $\alpha$ value of 0.88 , and the Kannada version of COMOT-15 had an $\alpha$ value of 0.82 , suggesting good internal consistency. The subscales of COMOT15 also showed good internal consistency, with $\alpha$ values of 0.83 for ear symptoms, 0.82 for hearing function, and 0.85 for mental health. The item-total correlation for COMQ-12 ranged from 0.44 to 0.81 , and for COMOT-15, it ranged from 0.36 to 0.75. The results of Cronbach's $\alpha$ and the item-total correlation are depicted in - Table 1. The complete translated questionnaires in Kannada and the English questionnaires are provided in -Supplementary material (online only).

Multiple regression analysis showed that the predictors explained $65 \%$ of the variance (coefficient of determination $\left.\left[\mathrm{R}^{2}\right]=0.65\right)$ for COMOT-15 scores, and $69 \%$ of the variance $\left(\mathrm{R}^{2}=0.69\right)$ for COMQ-12 scores. In addition, we found that PTA and onset of the hearing loss significantly $(p<0.05)$ predicted the scores. There was a significant positive correlation between PTA and COMOT-15 (correlation coefficient $[\mathrm{r}]=0.64, p<0.05)$ and COMQ-12 $(\mathrm{r}=0.69, p<0.05)$ scores. There was also a positive correlation between the onset of the hearing loss and COMOT-15 ( $\mathrm{r}=0.58, p<0.05)$ and COMQ-12 ( $\mathrm{r}=0.64, p<0.05)$ scores. Multiple regression also suggested that gender and was a poor predictor of COMOT-15 and COMQ-12 scores. The results of the multiple regression analyses are depicted in - Table 2.

\section{Discussion}

Health-related quality of life measurements in individuals with CSOM assesses the impact on overall health, social interaction and psychological status. It is essential to develop HRQOL measures in native languages so that they can be used on larger groups of the population. It is also important to develop questionnaires that are appropriate to the culture of the region. In addition, the social and health-related issues specific to that geographical location should be considered during the translation. Considering all of the aforementioned factors, the present study attempted to translate and validate COMOT-15 and COMQ-12 to the Kannada language. The translated versions were administered to a large group of the population with CSOM for validation. The results of the study showed that the translated versions of COMQ-12 and

Table 1 Cronbach's alpha and item correlation range values for the questionnaires and their subscales

\begin{tabular}{|l|l|l|l|l|l|}
\hline Measures & COMQ-12 & \multirow{2}{*}{ COMOT-15 } & \multicolumn{4}{l|}{ Subscales of COMOT-15 } \\
\cline { 3 - 6 } & & & Ear Symptoms & Hearing Function & Mental Health \\
\hline Cronbach's alpha & 0.88 & 0.82 & 0.83 & 0.82 & 0.85 \\
\hline Item correlation range & $0.44-0.81$ & $0.36-0.75$ & $0.41-0.77$ & $0.33-0.74$ & $0.43-0.78$ \\
\hline
\end{tabular}

Abbreviations: COMOT-15, Chronic Otitis Media Outcome Test-15; COMQ-12, Chronic Suppurative Otitis Media Questionnaire-12. 
Table 2 Results of the multiple regression analysis of COMOT-15 and COMQ-12 scores with pure tone average (PTA), onset of hearing loss, and gender as the predictors

\begin{tabular}{|c|c|c|c|c|c|c|}
\hline & \multicolumn{3}{|l|}{ COMOT-15 } & \multicolumn{3}{|l|}{ COMQ-12 } \\
\hline $\begin{array}{l}\text { Independent } \\
\text { variable }\end{array}$ & $\begin{array}{l}\text { Pearson's } \\
\text { Correlation }\end{array}$ & $\beta$ & $\mathrm{R}^{2}$ & $\begin{array}{l}\text { Pearson's } \\
\text { Correlation }\end{array}$ & $\beta$ & $\mathrm{R}^{2}$ \\
\hline Pure tone average & $0.64^{*}$ & $0.59^{*}$ & \multirow[t]{3}{*}{0.65} & $0.69^{*}$ & $0.57^{*}$ & \multirow[t]{3}{*}{0.69} \\
\hline Onset of hearing loss & $0.58^{*}$ & $0.41^{*}$ & & $0.64^{*}$ & $0.42^{*}$ & \\
\hline Gender & -0.03 & -0.03 & & -0.01 & -0.01 & \\
\hline
\end{tabular}

Abbreviations: COMOT-15, Chronic Otitis Media Outcome Test-15; COMQ-12, Chronic Suppurative Otitis Media Questionnaire-12; R², coefficient of determination.

Note: ${ }^{*} p<0.05$.

COMOT-15 and its subscales had good internal consistency and item-total correlations. This suggests that the test can be used to determine HRQOL in Kannada-speaking individuals with CSOM.

The results of the study also showed that Kannada-speaking individuals with CSOM show significant impairment in their health-related quality of life measures. The communication breakdown because of hearing loss affects their social interactions and hampers their professional lives. The ear-related symptoms, such as ear discharge, could further lead to social isolation, affecting the quality of life even more. ${ }^{6}$ The poor scores on the mental health scale show that CSOM also causes depression, anxiety and social withdrawal, which may reduce the quality of life. ${ }^{13}$ Thus, the study again highlights that, because of CSOM, there could be a restriction in communication that can impair daily life functioning.

The study also showed that the scores were poorer for a higher degree of hearing loss. Hearing loss plays an important role in determining the quality of life, especially in individuals with bilateral CSOM. This could be because of the withdrawal from social activities observed in such individuals. ${ }^{4,5}$ In addition, hearing loss restricts their ability to communicate, and affects their professional lives. This could lead to emotional problems in a few individuals with CSOM. ${ }^{12}$ The study also showed that the scores were poorer in individuals with a longer onset of the hearing loss. The longer restriction caused by hearing loss causes communication difficulty and impairs daily life functioning. ${ }^{9}$ Thus, audiologists and ear, nose and throat (ENT) specialists should incorporate the patient-centric approach by understanding the impairments in quality of life and providing the appropriate management. However, the study did not compare pre- and post-test scores after treatment because of a poor follow-up of the patients. Thus, further studies comparing pre- and post-treatment scores for the validation of the translated questionnaires are essential.

\section{Conclusions}

The present study attempted to translate and validate COMQ-12 and COMOT-15 in Kannada. The questionnaires were administered to a large group of individuals with CSOM for validation. The results of the study showed that the developed questionnaires have good internal consistency for measuring quality of life among individuals with CSOM. The study also showed that Kannada-speaking individuals with CSOM show significant impairment in their health-related quality of life measures. However, test-retest reliability of the developed questionnaire and its further validation are essential. Thus, HRQOL measures attempt to understand the problems of the individuals with CSOM from the patients' perspective and help clinicians provide the appropriate management.

Conflicts of Interest Statement

The authors report no conflicts of interest. The authors alone are responsible for the content and writing of the paper.

\section{References}

1 Nadol JB Jr, Staecker H, Gliklich RE. Outcomes assessment for chronic otitis media: the Chronic Ear Survey. Laryngoscope 2000; 110(3 Pt 3, S94)32-35

2 WHO. Chronic suppurative otitis media Burden of Illness and Management Options. 2004 [cited 2016 Nov 28]; Available from: http://www.who.int/pbd/publications/Chronicsuppurativeotitis_media.pdf

3 Varni JW, Burwinkle TM, Lane MM. Health-related quality of life measurement in pediatric clinical practice: an appraisal and precept for future research and application. Health Qual Life Outcomes 2005;3:34

4 Stewart MG, Coker NJ, Jenkins HA, Manolidis S, Bautista MH. Outcomes and quality of life in conductive hearing loss. Otolaryngol Head Neck Surg 2000;123(05):527-532

5 Kirkim G, Serbetcioglu B, Odabasi O, Mutlu B. Hearing loss and communication difficulty in the elderly. Mediterr J Otol. 2007; 3:126-132

6 Demir UL, Akyildiz MY, Alpay M. The Factors which Affect Disease-Specific Quality of Life in Patients with Chronic Otitis Media. Int Adv Otol. 2012;8(03):371-378

7 Phillips JS, Haggard M, Yung M. A new health-related quality of life measure for active chronic otitis media (COMQ-12): development and initial validation. Otol Neurotol 2014;35(03):454-458

8 Baumann I, Kurpiers B, Plinkert PK, Praetorius M. Development and validation of the Chronic Otitis Media Outcome Test 15 (COMOT-15). Measurement of health-related quality of life in patients with chronic otitis media. HNO 2009;57(09): 889-895

9 Baumann I, Gerendas B, Plinkert PK, Praetorius M. General and disease-specific quality of life in patients with chronic 
112 Development and Administration of COMQ-12 and COMOT-15 in Kannada Prabhu et al.

suppurative otitis media-a prospective study. Health Qual Life Outcomes. BioMed Central 2011;9:48

10 Bradley C. Translation of questionnaires for use in different languages and cultures. In: Bradley C, editor. Handbook of Psychology and Diabetes. Switzerland: Harwood; 1994. p. 43-55

11 Clark JG. Uses and abuses of hearing loss classification. ASHA 1981;23(07):493-500
12 Lee J, Witsell DL, Dolor RJ, Stinnett S, Hannley M. Quality of life of patients with otitis media and caregivers: a multicenter study. Laryngoscope 2006;116(10):1798-1804

13 Korsten-Meijer AGW, Wit HP, Albers FWJ. Evaluation of the relation between audiometric and psychometric measures of hearing after tympanoplasty. Eur Arch Otorhinolaryngol 2006; 263(03):256-262 